\title{
The Optimized Research Framework of the Road Traffic Delivery Service Supporting System Based on Dynamic Capability
}

\author{
SUN Hao \\ School of Economics and Management \\ Beijing University of Technology \\ YunXingYu Technology Service Co., Ltd, \\ Beijing, China \\ ssd1929@gmail.com
}

\author{
RUAN Pingnan \\ School of Economics and Management \\ Beijing University of Technology \\ Beijing, China
}

\author{
LIU Xiaoyan \\ School of Economics and Management \\ Beijing University of Technology \\ Beijing, China
}

\begin{abstract}
The delivery security system is the supporting system for guaranteeing the delivery system running, it has double attribute of public benefit and economics. This paper has used this as a starting point to analyze three main problems that the traffic delivery service supporting system is facing. We propose a dynamic capability improvement on both economy and society. We build the research framework for the traffic delivery service supporting system on three aspects of perceiving opportunity and threats capability, capturing opportunities capability and threats management capability.
\end{abstract}

Keywords-The traffic delivery service supporting system; Dynamic capability; Resource allocation

\section{INTRODUCTION}

Nowadays road traffic plays an important role in the economic development, it forms the traffic economy which is based on the main traffic trunk road in theory. In practice, it becomes the carrier of logistics and passenger flow between areas and cities, and plays the important role in the realization of regional economic harmonious development. It will influence the development speed and efficiency of national economy whether the traffic system can work well or not. To meet the needs of the economic development, the highway mileage increases by years. It is 4008229 kilometers in 2010, whose classified highway mileage is 3304709 kilometers; expressway mileage is 74113 kilometers [1]. In comparison, the national highway mileage is 4356218 kilometers in 2013, whose classified highway mileage is 3755567 kilometers, expressway mileage is 104438 kilometers. The national highway mileage in 2013 has increased by 8.7\% compared with 2010, and the classified highway mileage has increased by $13.6 \%$, the expressway mileage has increased by $40.9 \%$.

Road traffic network serves economic network, once the road traffic network with relative stability has been built up, the new road is just an extension of the original road. However, the economic network has the dynamic character because of the development of national policy and local economy, which can make the road traffic network and economic network do not match, and also make the road traffic network can't support the economic network. As a result, the importance of road traffic for national economy will be nothing. To relief the mismatch problem between the two networks, we can improve the carrying efficiency of the road traffic network.

\section{THE ROAD TRAFFIC SYSTEM AND THE TRAFFIC DELIVERY SERVICE SUPPORTING SYSTEM}

To improve the carrying efficiency of the road traffic network, we should know the component of road traffic system. As shown in Fig. 2, road traffic system consists of four elements that people, car, road and environment. And the elements of people including the driver, passengers and pedestrians; car is the carrier on the road; and they constitute the traffic delivery system together. Road include infrastructure design, infrastructure construction and infrastructure operation. And infrastructure construction includes road facilities and electromechanical system, electromechanical system is also an important sub-system in the ITS. They serve the traffic delivery system together, and are named the service system of traffic delivery. The role of this system is to guarantee the traffic delivery system running smoothly so that we can use it to serve the national economy. Infrastructure operation also includes two parts the road facilities maintenance and electromechanical system operation. They serve the traffic delivery system together and support the stable performance of the traffic delivery system to guarantee the system smoothly, and named as traffic delivery service supporting system. Environment mainly refers to the natural environment, such as air, noise etc.

In the whole road traffic system, the road facilities have long construction cycle, the road network won't be changed after completing the construction. In the case of constructing strictly accordance with standard and normal use, the system’s 
structure has good stability performance so that the road facilities aren't easy to be damaged. Hence we should make the work focus on the electromechanical system, and the electromechanical system O\&M.

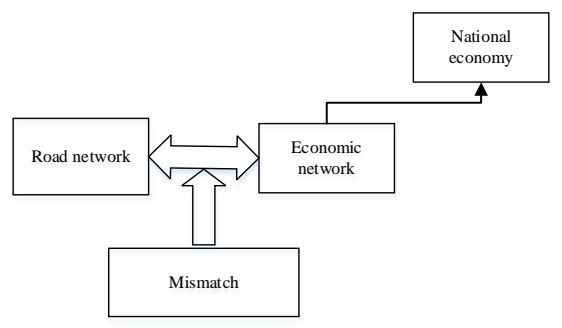

Fig. 1. The relationship between road network and economic network.

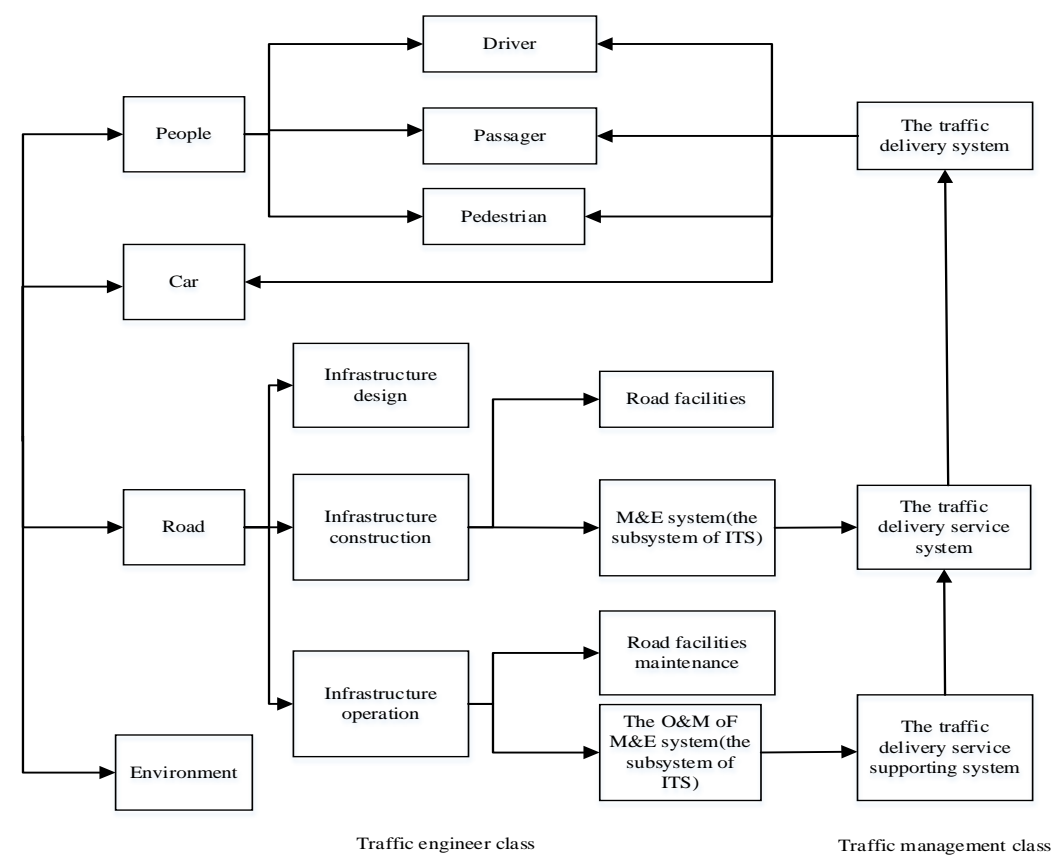

Fig. 2. Traffic network

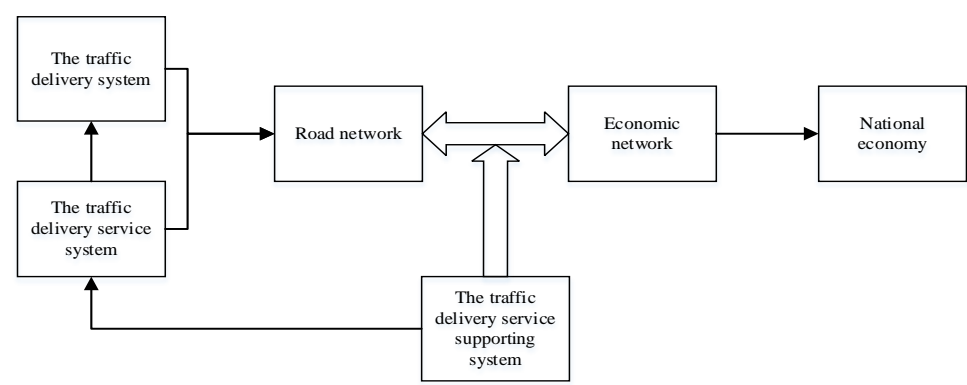

Fig. 3. The relationship between the traffic delivery service supporting system and the traffic delivery network and the economic network

In conclusion, the traffic delivery service supporting system refers the general name that aims to guarantee the traffic going smoothly and provides the traffic delivery system services, such as operation, maintenance etc.

As Fig. 3 shows, the economic network has the dynamic character because of the development of national policy and local economy, which makes the road traffic network and economic network do not match, so it becomes a main problem that how to use the traffic delivery service supporting system to minimize the damage which is caused by the mismatch. Hence only we programing the resource allocation of the traffic delivery service supporting system reasonably, can we relief the negative effects which are caused by the heterogeneous between the economy network and the road traffic network, improve the carrying efficiency of the road traffic network, thus guarantee the well-developed of national economy. 


\section{THE PREDICAMENT OF THE TRAFFIC DELIVERY SERVICE SUPPORTING SYSTEM}

Now the research on the traffic delivery service supporting system mainly focus on transportation security and traffic combat readiness, etc. [2] Some Scholars think that the system should include the following four aspects: policy and regulation guarantee, system guarantee, infrastructure construction guarantee, technical guarantee [3]. This article mainly focuses on the involved technical and facilities guarantee in the carrying service, and how to manage them. At present, there mainly exist the following problems:

(1) We are overly concerned about economic attribute and ignored the social attribute during the resource allocation. Take highway for example, monitoring equipment hasn't daily operational service in some areas, only when the equipment are damaged and has serious impact on work quality will be repaired. In the whole system crashes, charging system is priority. The traffic delivery service supporting system serves the traffic delivery system, and the traffic delivery system aims to make the traffic smoothly, so the traffic delivery service supporting system should guarantee the public welfare of smooth passage and enterprise's economy attribute. Traditional research from economic perspective aims to improve the system for the purpose of efficiency. But it is obviously contradictory between the efficiency and passage guarantee. So it becomes the key problem that how to coordinate the relationship between the two to improve the efficiency based on the passage guarantee.

(2) Capability to adapt the change of external environment becomes lower, and the operation energy efficiency of the service supporting system is lag behind. With the development of the economy, vehicle flow network will evolve constantly, but the road traffic network won't be changed after completing the construction, so it is more stable than vehicle flow network, therefore it make the two network do not match and damage the smoothly passage. The delivery service supporting system needs to relief the difficult position caused by the mismatch between the two network through resource allocation, now the resource allocating mode of the delivery service supporting system is based on decision model, it exits serious lag. As a result, it occurs other problem that how to quickly make the response for the changes of external environment and dynamically obtain the environment requirement to improve the service efficiency.

(3) The core of the delivery service supporting system optimizing is about resource allocation; resource allocation is not reasonable and results in an inefficient use of resources. The main reason is that the demand prediction mainly uses statistical tools for data analysis and uses regression analysis for data fitting, although we can find part regular characteristics of demands in this way, but it can only be used for analyzing trends when the change of external environment is not obviously. And when the updating equipment and the kinds of equipment are large, the prediction outcomes will be inaccurately. The quantity of equipment will be influenced by the changing of highway traffic mileage and technology, etc. And it also can be influenced by the factors, such as usage, climate and link attributes, etc. Thus the fault prediction which is only based on few factors in the statistics can't reflect the characteristics of all sections. Human resource forecasting takes all the staff as homogeneous group without considering the different knowledge characters between them, the different complexity between maintenance tasks and different requirement characters for each person. Therefore if we make the resources allocating under those conditions, it is obviously that allocation optimization can't be achieved.

\section{SUMMARY OF DYNAMIC CAPABILITIES}

On the basis of evolutionary economics, Teece had proposed the concept of dynamic capabilities on the Strategic Management Journal in 1997, he pointed out the capabilities for a company to integrate, build or reset the competition capability of external and internal to adapt the rapidly changing external environment, and "dynamic" refers that the enterprise must have the adaptation about the turbulent external environment to adapt the changing market; "capabilities” refers that the enterprise should improve their own capability(such as integrate, rebuild the organizational skills or resource of external and internal) to satisfy the requirement of changing environment, that is to say, the capability of driving and changing existing capabilities. Dynamic capabilities emphasis that inner-enterprise should adapt the changing environment dynamically and combine the core capabilities and dynamic capabilities to generate the dynamic core capabilities for enterprises.

Jiang Jihai proposed the capability of perceiving, learning, integrating and innovating. In the respect of cultivating the perceiving capability, enterprises should strengthen the process of scanning, monitoring, forecasting and evaluating the changes of internal and external environment, perceive the changing trend of customer, Industry and competition, build the discernment of enterprises and management team, strengthen their innovative awareness, arouse the potential of employees actively, and guide the employees in different levels to pay attention on new technology, new markets and new business models. In the respect of cultivating the learning capability, enterprises should strengthen their capability of knowledge transferring, technology learning and knowledge management, motivate the learning motivation of knowledge worker, and cultivate thick atmosphere of learning. In the respect of cultivating the integrating capability, enterprises should enhance the combination of existing knowledge and the new knowledge which is obtained by perceiving and learning, strengthen the integration of resources, conventions, operational capability and core capability between the internal and external. In the respect of cultivating the innovation capability, enterprises should pay attention to the innovation of business models, enhance the investment of R\&D, and take the collaborative innovation openly and disruptive innovation seriously [4].

Teece discomposed “dynamic capability” into different parts, such as capability of realizing and specifying the chances and threats, capability of seizing the opportunities, and capability of sustaining competitiveness by strengthening, combining or reconfigure as needed the tangible and intangible assets of enterprises. Wu discomposed "dynamic capability" into resource integration capability, resource reconfiguration 
capability, learning capability, and the capability of making response to the changing market[5], Dong Baobao et al. discomposed "dynamic capability" into five dimension of resource integration capability, resource reconfiguration capability, learning capability, adaptability and innovation capability[6]. Drejer thought the factor influenced the development can be boiled down to technological change, customer requirement and organizational learning; what's more, he combined all the three factors and built a model. This model takes capability developing as a force field which is formed by the interconnected driving forces and all the three factors is exactly what the interconnected driving forces refer to. Knowledge sharing and knowledge creation greatly depend on the organizational learning, the interaction of environment and enterprises is done though the senior managers' cognition and decision which plays the decisive role in the depth and breadth of organizational learning. Therefore, organizational learning is the foundation of building and improving the core capability for the enterprise and an essential way to obtain the sustaining competitive advantages.

\section{The Optimized RESEARCH FramewORK OF THE DELIVERY SERVICE SUPPORTING SYSTEM BASED ON DYNAMIC CAPABILITY}

The resource in the traffic delivery service supporting system includes tangible resource, such as human resource, equipment resource, etc. and intangible resource, such as innovation resource, knowledge resource, information resource, etc. In this paper, in consideration of the traffic delivery service supporting system's characteristics ,and the intangible resource adhere to tangible resource so that it depends on the human resource, therefore we mainly take equipment resource and human resource into consideration when research on the optimizing of resource allocation. Teece et al. originally proposed the concept that the dynamic capability means the capability to change capabilities, and defined the dynamic capability as the capabilities for a company to integrate, build or reset the competition capability of external and internal to adapt the rapidly changing external environment. Obtained the dynamical capability means that the enterprise possesses new usage knowledge about related resource, new ways for resource integration, that is to say, enterprise can stimulate potential service which is underlying the original resource, obtain the resource service which is possessed by other enterprises and new way to integrate and configure resource in harmony.

The traffic delivery service supporting system aims to keep the traffic smoothly, so the resource allocation in this system should be optimized based on this target, that is to say, the resource allocation target has a dual property: Sociability and Economy. Although the traditional resource allocation and optimizing model which focused on fairness and efficiency can solve the efficiency problem that belongs to economy attribute, it can't solve the social problem, so we need to find a new solution to guarantee the dual property. The dynamic capability theory is proposed by the strategy management researchers based on the RBV, dynamic capability is enterprises' higherorder abilities for obtaining the competitive advantages, it is also the ability to make quick response to the changing external environment and adapt it as soon as possible. The resource in the traffic delivery service supporting system is the core resource of traffic delivery service supporting enterprises. With the changing external technology conditions, the configuration rules and resource attribute transforming constantly. Therefore

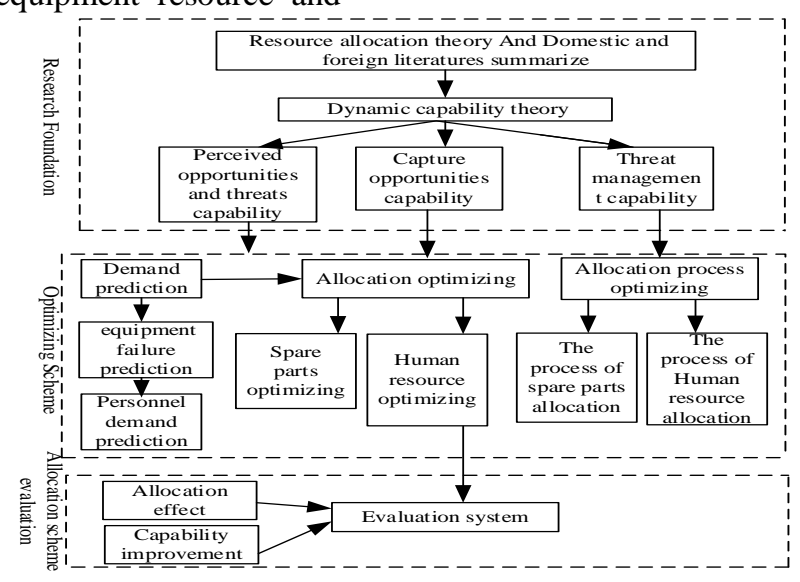

Fig. 4. Research framework

only thinking about the related resource allocation of the delivery service supporting system from resource-based view is not enough to solve the equipment updating problem and demand uncertainty caused by the changing external environment or technical changes. We should start by improving the dynamical resource allocation capability of delivery service supporting enterprises and study on the resource allocation. So this paper will take the dynamic capability as the logical starting point to give an optimized resource allocation proposal which can deal with the external uncertain demand and improve capability of data integration and knowledge integration for delivery service supporting enterprises. In consideration of the view of dynamic capability theory, dynamic capability includes three levels as follow: Perceived opportunities and threats, Capture opportunities, Threat management. And it will build the optimized resource allocation proposal for the delivery service supporting system in accordance with the three levels: Perceived opportunities and threats by predicting equipment failure, Capture opportunities by building optimized resource allocation model 
of human resource and spare parts resource, Manage threats by optimizing the process of personnel scheduling and spare parts allocating.

\section{A. Perceived opportunities and threats-- Machine condition assessment and prediction}

The resource demand in the delivery service supporting system mainly focuses on the maintenance service and repair service of M\&E equipment. Service supporting work aims at three parts: firstly, capture the machine conditions quickly and make reasonable maintenance plan; secondly, make prediction for the possible system failure and reduce the incidence of fault; thirdly, response the failure quickly and repair it at once. Perceived opportunities and threats is the capability of quickly identifying the changes of enterprise external environment. And the service features of the delivery service supporting enterprise determine the core task of perceiving opportunities and threats is to find out equipment failure in time and even realize fault early warning. The realization of the above goals is on the basis of scientific prediction. The main idea of demand prediction which is around the delivery service supporting system's core work is: make maintenance plan in accordance to equipment working condition, analyze the associations between the failure and the working condition, maintenance condition and usage of the equipment based on the historical data, analyze the failure probability refer to historical failure data. So the research content of this section includes: maintain demand prediction, build equipment health indicator to evaluate equipment usage and make the demand prediction based on the health indicator, repair demand prediction.

\section{B. Capture opportunities-resource allocation optimizing}

Enterprise should use its existing resource to maintain and repair equipment in the condition of failure prediction or a failure happens. The dynamic capability view refers to the resource allocation, it points out that enterprises should establish new advantages in accordance to changes of external environment. Delivery service supporting enterprises dynamic capability is shown in quickly response to equipment failure. But the resource allocation under the dynamic capability framework will be done for the human resource and spare parts combining demand prediction and the possibility of equipment failure. It contains the following content: first, the allocation schemes for spare parts stock. It mainly builds the reference stock scheme and the spare parts' resource allocation stock scheme of different service departments or subordinate maintenance group; second, personnel allocation scheme. It builds the complex network model above the personnel, knowledge and tasks in accordance to the possible failure and their types. And it also builds the personnel allocation model based on task demand through organizational learning theory, knowledge management theory and simulation.

\section{Threats management-resource allocation process optimizing}

Threats management mainly orients to the emergency management of outburst failure. Emergency management aims to response the demand quickly, which wants to minimize the repair time through allocating related resource. This section mainly researches on the following two aspects: firstly, the quick equipment allocation process attempts to measure the relationship strength and priority for roles dependence, device dependence and facilities dependence, and use the structure matrix to plan the allocation location and process of spare parts reasonably. Secondly, the human resource allocation process optimizing makes the personnel allocation selection in accordance to the failure possibility and personnel saturation of maintenance points using the way of knowledge map and data mining to solve the personnel shortage problem.

\section{The resource allocation evaluation based on the dynamic capability}

This section mainly researches on how to evaluate the effect of the resource allocation. The resource allocation evaluation includes two dimensions: one is allocation effect, and another is capability improvement. This paper will build multi-objective comprehensive evaluation model around above two dimensions and apply the HOQ to the analysis of dynamic capability, it discusses the indicator of dynamic capability evaluation and its autocorrelation and the autocorrelation of resource allocation factors. It also builds the optimizing model of dynamic capability and resource allocation cost on the basis of quality function, which takes the dynamic capability as the objective function and takes the resource allocation as cost function.

\section{CONCLUSION}

With the promotion and application of information technologies, such as ETC, EPC network, etc. The delivery system's installation number M\&E equipment increased dramatically in recent years, the equipment technologies has made the unprecedented challenge for the delivery service supporting system. In the view of dynamic capability and taking the data as the core, we propose capability to improve consider about resource allocation. And In this way, we have broken the traditional research model which is focus on economic efficiency, solved the irrational resource allocation caused by pursuit the short-term benefits, and realized the dynamic allocation optimizing with the adaptive capability.

\section{REFERENCES}

[1] Ministry of Transport of the People's Republic of China. China Transport Statistic Yearbook, 2013

[2] Zhao Zhanping. Build the Civil-Military Integrated comprehensive transportation support system, comprehensive transportation, 2007

[3] Hao Xiaohui, Guo Lin. The transportation support system research in the process of urbanization [J], Shandong Transportation technology. 2008, 1

[4] Jiang Jihai, Liu Min. An Empirical Research on Dynamic Capabilities Deconstruction and their Relationship with Competitive Advantage [J]. Science Research Management, 2014, 08: 75-82

[5] Lei-Yu Wu. Entrepreneurial resources, dynamic capabilities and start-up performance of Taiwan's high-tech firms [J]. Journal of Business Research, 2007, 60: 549-555

[6] Dong Baoao, Ge Baoshan. Research on the Relationship between Resource Integration Process of New Venture and Dynamic Capability [J]. Science Research Management, 2012, 02: 10 